\title{
Postures professionnelles concernant les trans- actions sexuelles impliquant des jeunes : entre souci de (sur)protection et accompagnement de la socialisation sexuelle
}

Résumé:À partir des données récoltées dans le cadre d'une recherche sur les transactions sexuelles impliquant des jeunes, cet article s'intéresse aux postures des professionnel-le·s du travail social et de la santé face à ces pratiques. Il met en lumière différentes représentations de la place donnée à l'éducation sexuelle, entendue comme toute forme d'intervention liée à la sexualité. Entre postures de protection et d'accompagnement, peu sont celles et ceux en travail social qui se sentent légitimes d'aborder les questions d'éducation sexuelle au sein de leur travail socio-éducatif. Or, les jeunes en sont demandeuses et demandeurs. Il en découle l'importance d'offrir à ces professionnel-le·s des formations appropriées afin de mieux intégrer l'éducation sexuelle plus spécifiquement dans le travail social auprès des jeunes. Mots-clés: travail social, éducation sexuelle, jeunes, sexualité, santé sexuelle

\section{Professional dispositions in regard to sexual transactions of youth: Balancing (over)protection and support for sexual socialisation}

Summary: Based on data collected in a research on sexual transactions of youth, this article discusses the attitudes and professional dispositions of health and social workers in regard to these practices. It sheds light on the different scope given to sexual education, understood as any form of intervention related to sexuality. Having to balance professional commitments of protecting and supporting youth, very few social workers feel legitimate to address concerns of sexual education in their engagements with youth. However, young people need such engagements. It is therefore important to offer to social workers appropriate training in order to better integrate sex education into social work with youth.

Keywords: social work, sex education, youth, sexuality, sexual health 
On fait quoi? De l'éducation sexuelle [...]? On a plein de questions sur le feu: ce qu'on peut dire, ce qu'on ne peut pas dire... à quel âge mieux vaut de commencer? Avant 12 ans, non, car c'est de la compétence de la famille. Après, ben, il est déjà trop tard. (Matteo, psychologue, foyer pour jeunes en difficulté, Tessin')

Ce témoignage, recueilli dans le cadre de la recherche «Sexe, relations... et toi?» (Colombo et al., 2017a), illustre les doutes et questionnements que peut soulever l'éducation sexuelle dans le travail social auprès des jeunes. Plus précisément, cette étude, financée par la Fondation Oak, trouve son origine dans des inquiétudes exprimées par quelques professionnel-le·s se sentant démuni.e.s face à des pratiques associant échange et sexualité observées chez certain·e·s jeunes, relayées par la section Child Abuse de la Fondation Oak. Cette étude menée en Suisse de 2015 à 2017 en trois langues (français, allemand, italien) s'est intéressée d'une part aux représentations et expériences des jeunes femmes et hommes âgés de 14 à 25 ans concernant des transactions sexuelles et, d'autre part, aux représentations sociales des professionnel-le-s socio-sanitaires ${ }^{2}$ sur ce même sujet. Les transactions sexuelles désignent ici toute expérience d'ordre sexuel associée à un échange financier, matériel et/ou symbolique, par exemple (se faire) offrir un verre ou de l'argent, être accepté·e dans un groupe ou encore s'échanger des photos érotiques.

Cet article se base uniquement sur les entretiens réalisés auprès des professionnel-le.s. Pour la plupart non spécialistes de l'éducation sexuelle, elles et ils sont confrontés à différentes manifestations liées à la vie sexuelle de ces jeunes, que ce soit par des blagues sexistes ou homophobes, par des questions liées à des habillements ou des comportements jugés trop "sexy», ou par des situations plus complexes telles que les transactions sexuelles, sans toujours savoir comment réagir, comme en témoignent les propos de Matteo cités en introduction. Psychologue, responsable d'un foyer qui accueille des jeunes en rupture familiale situé au Tessin, cet intervenant fait part, dans son récit, de son envie de bien accomplir sa mission socio-éducative tout en soulevant des questions en lien avec l'éducation sexuelle telles que le contenu des messages, le moment de l'intervention et la part de responsabilité de la famille, de l'école et du réseau professionnel du travail social.

Cet article analyse les postures professionnelles de ces intervenant.e.s face à la sexualité d'ordre transactionnel dans le travail socio-éducatif auprès des jeunes. La première section contextualise l'analyse pré- 
sentée dans cet article par rapport aux recherches existantes liant travail social et sexualité. La deuxième section décrit la démarche méthodologique de l'enquête dont sont issues les données présentées. Les deux dernières sections présentent deux postures professionnelles qui se dégagent de l'analyse, que nous avons nommées la posture de protection et celle d'accompagnement. En conclusion, nous mettons ces résultats en discussion avec les résultats des entretiens réalisés auprès des jeunes.

\section{Travail social et éducation sexuelle: d'une approche biomédicale à une approche holistique?}

La littérature montre que si le processus historique et sociologique de médicalisation de la sexualité a autorisé une lecture en termes médicaux des problèmes liés à la sexualité, il a eu comme conséquence d'éclipser la dimension sociale de la sexualité (Giami, 2009). Ce n'est qu'à partir du concept de santé sexuelle que cette dimension a été revalorisée. Le concept de santé sexuelle, forgé en 1974 lors d'une conférence de l'Organisation mondiale de la Santé (OMS), désigne un «état de bien-être physique, mental et social dans le domaine de la sexualité »3 ; il rend légitime autant l'idée de l'exercice d'une vie sexuelle non reproductive que de son importance pour le bien-être et la santé. Dans la mesure où la santé sexuelle n'est pas réduite au traitement ou à l'absence de maladies ni à la procréation, elle se détache de l'univers de la vénérologie et de la gynécologie pour s’approcher de celui du social (Giami, 2009).

Dans cette perspective, une théorie proposant une approche holistique de l'éducation sexuelle a été proposée dans les années 2000 par plusieurs organisations internationales telles que l'OMS (OMS Bureau régional pour l'Europe/BZgA, 2013) et a été reprise, en Suisse, par Santé Sexuelle Suisse, l'organisation faîtière des centres de santé sexuelle (Charmillot \& Jacot-Descombes, 2018). En s'appuyant sur le concept de santé sexuelle, cette approche holistique met l'accent sur les différentes dimensions de la sexualité (biologique, médicale, affective, sociale, juridique, etc.), considérées comme complémentaires, en insistant particulièrement sur l'encouragement d'une éducation sexuelle basée sur les droits sexuels. Une telle éducation implique d'une part, la transmission des connaissances, mais surtout le renforcement des compétences sociales et psychosociales des individus et, d'autre part, le partage des tâches entre la famille et le réseau professionnel de santé et de travail social (Charmillot \& Jacot-Descombes, 2018). 
Cette approche suppose donc que les professionnel-le·s du travail social participent (ou sont censé·e·s participer) à l'éducation sexuelle des personnes qu'elles et ils accompagnent. Si elles et ils ont un rôle à jouer dans cette tâche, la littérature montre qu'il n'est pas, pour autant, évident de faire de la sexualité un sujet important dans leurs interventions et encore moins d'adopter une approche d'éducation sexuelle holistique. Ce qui ne paraît pas étonnant si on se penche sur la place de la sexualité dans le cursus de formation de ces corps de métier. À cet égard, la littérature met en évidence deux aspects. Tout d'abord, en raison de la présence des tabous (Merotte, 2012), d'une croyance religieuse ou d'un manque de connaissances et de formation (Schaub et al., 2017), les professionnel-le·s abordent difficilement les thèmes liés à la sexualité sous un angle holistique. Les principales préoccupations des travailleuses et travailleurs sociaux concernent les abus, la violence sexuelle, les infections sexuellement transmissibles (IST) et le VIH (Dodd \& Tolman, 2017). Ensuite, au niveau des programmes d'étude de travail social, ce sujet semble recevoir peu d'attention, comme le montre par exemple Rowntree (2014) pour le plan d'études australien. Lorsque le sujet fait partie du cursus de formation, il est traité à partir d'expériences négatives de groupes minoritaires (LGBT, homophobie, etc.) (Morton et al., 2013). En Suisse romande, le nouveau Plan d'étude cadre du Bachelor en travail social ne contemple que très timidement les questions de sexualité (HES-SO, 2020). Pourtant, quelques études insistent d'une part, sur l'importance d'intégrer et/ou renforcer, en tant qu'axe critique du travail social, la sexualité dans la formation (McCave et al., 2014). D’autre part, elles soulignent le besoin d'approcher ce sujet dans une perspective holistique (Dodd \& Tolman, 2017).

Alors que la thématique de la prise en compte de la sexualité dans le cadre de la relation soignante a fait l'objet de plusieurs publications scientifiques ${ }^{4}$, c'est moins le cas pour ce qui concerne la relation socio-éducative. Deux constats émanent des études consultées dans ce domaine. Premièrement, elles montrent la prédominance autant dans les représentations que dans l'intervention d'une approche biomédicale qui réduit la sexualité à ses dimensions physiques abordées notamment sous la perspective des risques. Tel que le souligne Moulin (2007), la sexualité est avant tout pensée comme un problème qui se manifeste par différents dangers: l'érotisation des relations, les conduites de harcèlements sexuels, les accusations de pédophilie, les abus sexuels, etc. Les actions se limitent, selon les contextes institutionnels, à la prévention des IST et/ou l'encouragement de l'usage d'un moyen de contraception (Ménoreau \& Dupras, 2014). Une telle 
approche donne peu ou pas de place aux enjeux relationnels inhérents à la vie affective et sexuelle des jeunes, alors que ces aspects sont au centre de leurs questionnements (Amsellem-Mainguy \& Dumollard, 2016) et qu'elles et ils souhaiteraient en pouvoir en parler plus souvent avec les intervenant·e·s (Côté et al., 2015).

Deuxièmement, certaines études soulignent le rôle des normes de genre et de sexualité. Profondément intériorisées par les professionnel-le·s, ces normes influencent leurs postures, qui peuvent même contribuer à renforcer l'ordre du genre (Joël, 2014 ; Amsellem-Mainguy \& Dumollard, 2016). Ces études montrent que la plupart de ces personnes se basent sur une conception bicatégorielle du sexe et du genre et que leurs interventions sont fortement imprégnées par la représentation d'une sexualité masculine prépondérante. Alors que les garçons sont présentés comme hyperactifs sexuellement, hétérosexuels et dominants, les filles, quant à elles, apparaissent comme soumises, sérieuses et à préserver.

Or, les études existantes ne se concentrent pas forcément sur la population juvénile et ne portent pas spécifiquement sur la place accordée à l'éducation sexuelle dans l'accompagnement des jeunes (Moulin, 2007). Celles qui s'intéressent à la sexualité juvénile ciblent des groupes particuliers, tels que les personnes détenues (Joël, 2014; Amsellem-Mainguy \& Dumollard, 2016), les jeunes de la rue (Côté et al., 2015) ou ayant une déficience intellectuelle (Ménoreau \& Dupras, 2014).

Tout en rejoignantles constats deces études, cet articleles complète en analysant les postures professionnelles relatives à la sexualité d'ordre transactionnel dans le travail socio-éducatif auprès des jeunes, au-delà de la diversité des problématiques vécues (jeunes en difficulté familiales et scolaires vivant dans des foyers, jeunes fréquentant des centres d'animation socio-culturels, jeunes des écoles, etc. $)^{5}$.

\section{Méthodologie}

Issu.e.s de différents domaines du travail social, les professionnel-le·s interrogé·e·s dans le cadre de notre étude interviennent auprès des jeunes, hétérosexuel-le·s et/ou homosexuel-le·s et ont toutes et tous été confrontés, directement ou indirectement, au moins une fois, à des situations d'échanges sexuels, ou se sentaient concernés par la thématique de la sexualité des jeunes.

Sur une centaine de personnes de différentes régions linguistiques contactées dans le cadre de cette étude qualitative, 34 ont pu être interrogées (cf. annexe 1) par le biais de focus groups, c'est-à-dire des entretiens 
collectifs basés sur des discussions thématiques permettant à chacune et chacun de s'exprimer autour de matières définies au préalable. Cette méthode permet d'aller au-delà des témoignages individuels pour repérer des représentations communes quant aux thèmes évoqués (Krueger, 1998).

Cinq focus groups constitués de 6 à 8 personnes ont été réalisés : 3 en Suisse romande, 1 en Suisse allemande et 1 en Suisse italienne. La majorité des participant.e.s possèdent une formation sociale (éducatrices et éducateurs sociaux, assistantes et assistants sociaux, animatrices et animateurs socioculturels, psychologues); d'autres une formation en santé (infirmières et infirmiers scolaires) et quelques-un·e·s bénéficient d'un diplôme postgrade en éducation ou conseil en santé sexuelle. Ces entretiens ont été enregistrés, retranscrits et traduits en français lorsque nécessaire.

L'objectif de ces groupes de discussion était de saisir les représentations qu'ont ces intervenant.e.s des transactions sexuelles, ainsi que les pratiques d'intervention qui en découlent. Les représentations sociales sont définies comme une forme de connaissance, socialement élaborée et partagée, ayant une visée pratique et concourant à la construction d'une réalité commune à un ensemble social. Également désignée comme «savoir de sens commun" ou encore "savoir naïf», "naturel», cette forme de connaissance est distinguée, entre autres, de la connaissance scientifique (Jodelet, 1989, p. 53)

Les représentations constituent une grille de lecture et de décodage de la réalité, autrement dit, elles nous permettent, d'une part, d'expliquer la manière dont un individu ou un groupe d'individus lit la réalité dans laquelle il évolue et, d'autre part, d'interpréter la réalité qui régit les relations des personnes à leur environnement physique et social. Dans ce sens, les représentions sociales « orientent et organisent les conduites et les communications sociales» (Jodelet, 1989, p. 53).

Lors de ces entretiens de groupe, nous nous sommes intéressées plus précisément à la façon dont ces représentations influencent les postures des professionnel-le·s relatives à la sexualité des jeunes, notamment lorsque que ces dernières et derniers sont impliqués dans des transactions sexuelles. Ces dernières sont définies comme des expériences d'ordre sexuel associées à un échange financier, matériel et/ou symbolique. Cette définition, inspirée de celle de Broqua et Deschamps (2014), privilégie d'une part, le terme "d'expériences sexuelles" (concept qui renvoie à une diversité de pratiques sexuelles mais aussi aux sens donnés par les actrices ou acteurs) et, d'autre part, celui d'échange (au lieu de rétribution pour souligner le caractère dynamique et rarement unilatéral de ces transactions). 
Cette définition s’inscrit dans l'approche des "sexualités négociées» proposée par Combessie et Mayer (2013), qui, tout en reprenant l'idée d'un continuum en transactions marchandes et non marchandes (Zelizer, 2001 ; Tabet, 2004), intègre une diversité de types d'échange (économies financière, affective et de la reconnaissance). Lors de la récolte de données, la formulation «expériences sexuelles en échange de quelque chose» a été privilégiée, afin de laisser le plus de place possible à la subjectivité des personnes enquêtées.

Les données récoltées ont été analysées à l'aide d'une grille d'analyse des repères normatifs composant les représentations des actrices et acteurs, développée par Parazelli et ses collègues (Colombo et al., 2016), en s'inspirant des travaux de Karsz (2004). Ces repères, qui permettent d'appréhender le sens d'un comportement selon le point de vue d'un individu qui l'exerce sont de trois ordres: cognitif (comment les répondant.e.s expliquent-elles ou ils les pratiques de transactions sexuelles et se représentent-elles ou ils les jeunes qui les pratiquent?) ; éthique (comment légitiment-elles ou ils leur posture professionnelle?) et politique (quelles stratégies d'intervention privilégient-elles ou ils?).

Dans la suite de l'article, afin d'illustrer notre analyse, nous présenterons les propos des répondant.e.s par le prénom d'emprunt, suivi des indications suivantes: profession, poste occupé, lieu de travail et sa localisation selon la région linguistique. Étant donné la nature qualitative de l'enquête, ces caractéristiques ont moins été utilisées à des fins d'analyse que pour situer le contexte des propos des personnes interviewées.

L'analyse de ces entretiens met en lumière différentes représentations de la place donnée à l'éducation sexuelle, entendue comme toute forme d'intervention liée à la sexualité, dans leur travail social auprès de ce public. Pour certain·e·s, l'éducation sexuelle relève d'une action concurrentielle à celle des parents, se justifiant tout de même lorsque ceux ou celles-ci ne sont pas en mesure de l'assumer. Ce type de représentation s'inscrit plutôt dans une approche biomédicale qui considère la sexualité de manière compartimentée. Pour d'autres, il s'agit d'une responsabilité partagée entre le public et le privé, ce qui justifie une intervention importante dans le travail social auprès des jeunes. On retrouve ici une vision qui se rapproche plutôt d'une vision holistique de l'éducation sexuelle.

Ces représentations donnent lieu à des postures professionnelles s'inscrivant sur un continuum entre deux logiques: d'un côté, une posture que nous qualifions de protection (lorsque les parents échouent à leur tâche d'éducation sexuelle) et de l'autre, une posture que nous nommons 
d'accompagnement (comprise comme complémentaire à celle des parents). Précisons que ces logiques subjectives typifiées pour faciliter la compréhension se concrétisent cependant souvent de façon plus nuancée et complexe dans la réalité et peuvent changer dans le temps.

Dans la suite de l'article, nous analysons chacune de ces deux logiques selon la grille des repères normatifs présentée plus haut, en mettant en lumière les représentations de la sexualité liée aux transactions sexuelles, et des jeunes qui s'y engagent (repères cognitifs), les arguments qui légitiment ces postures professionnelles (repères éthiques) et les stratégies d'intervention qui en découlent (repères politiques).

\section{Protéger des jeunes face aux risques sexuels}

D'un côté du continuum, on trouve la posture professionnelle de protection. Les individus qui privilégient cette posture considèrent l'éducation sexuelle comme relevant de la responsabilité des parents. Dans cette perspective, c'est principalement aux parents qu'il revient d'assumer la tâche d'encadrement des jeunes dans les questions de sexualité. Toutefois, il leur semble légitime d'intervenir lorsqu'il y a nécessité de défendre les jeunes de situations qui leur portent préjudice et face auxquelles les familles ne sont pas en mesure de le faire. Dans cette logique, le rôle de ces personnes est de protéger ces jeunes face aux risques qu'elles ou ils encourent en s'engageant dans des transactions sexuelles. Celles-ci sont associées à des pratiques de prostitution, à la consommation d'alcool et des drogues et à des risques sexuels (non utilisation des moyens de protection/contraception) et/ou d'autodestruction (scarifications, tentatives de suicide). Le caractère problématique de la situation est souvent posé comme une évidence, non seulement en raison de sa nature déviante, mais également par son effet cumulatif (plusieurs pratiques simultanément) et son côté asymétrique (différences d'âge et de statut).

L'analyse montre que les jeunes femmes et hommes sont, à plusieurs reprises, qualifiés d'inconscients, immatures, irresponsables et victimes de pressions. Influençables et possédant une faible estime de soimême, ces jeunes seraient prêt.e·s à accepter de s'engager dans des relations où ils et surtout elles sont dominées ou abusées et/ou impliquées dans des situations de manipulation difficiles à gérer, comme en témoignent les propose de René, travailleur social et responsable d'un service communal de jeunesse dans un canton de Suisse romande. 
Où ça commence à devenir une sexualité entre guillemets "problématique» qui fait souffrir, en tout cas les gens qu'on côtoie, c'est surtout par rapport à des jeunes qui ont une très très faible estime de soi et qui sont prêts à tout pour être simplement aimés. Et puis ils ne savent pas très bien ce que ça veut dire "amour». La sexualité, c'est un peu la même chose. Et puis on peut se donner à n'importe qui. (René)

Cette logique considère les jeunes qui s'impliquent dans des transactions sexuelles comme des victimes d'une socialisation défaillante qui, comme le suggère René, ne les a pas suffisamment dotées des ressources nécessaires (par exemple distinguer ce qu'est l'amour) pour gérer de façon mature et responsable leurs relations sexuelles. Ces jeunes femmes et hommes (mais aussi leurs familles) sont considérés comme vulnérables, démunis des ressources et, n'étant pas en mesure de trouver des solutions par eux-mêmes et n'ayant pas de capacité de faire des choix raisonnables. Dans cette logique, là où le professionnel se considère alors comme le principal détenteur de ressources et son intervention se fonde sur une légitimation sanitaire, juridique et morale.

Une légitimation sanitaire, juridique et morale

Sans prétendre à l'exhaustivité, nous avons pu identifier trois types de légitimation de cette posture à partir des propos des personnes interrogées: sanitaire, juridique et morale. Ceux-ci s'articulent et peuvent prendre plus ou moins d'importance selon les missions institutionnelles.

L'argument sanitaire s'inscrit dans la promotion de la santé: le rôle des intervenant.e.s est de combler le manque de connaissances des jeunes en leur fournissant des informations d'ordre biologique et "technique» pour gérer les risques sexuels (dimensions santé/hygiène, notamment en termes de prévention des IST et grossesses non-désirées, de la pornographie, etc.).

Dans une optique de protection juridique, les jeunes femmes et hommes sont considérés comme ayant subi des préjudices d'une famille (et/ou d'autres institutions de socialisation) qui ne leur a pas donné les moyens d'affirmer leurs droits sur le plan sexuel, c'est-à-dire de mettre leurs limites et de se défendre des abus. Dans ce cas, le rôle de l'intervention sociale est d'apporter un soutien consistant à appliquer ou faire appliquer la loi et les différentes mesures de protection. Les propos de Danièle, assistante sociale dans un service en charge de la protection des mineur.e.s en Suisse romande, illustrent cette position. 
Moi, j'évolue dans un milieu où le cadre légal est très important [...] J'ai toujours comme habitude d'évaluer la situation en fonction d'un cadre légal: est-ce qu'il y a une victime, est-ce qu'il y a un coupable, quels sont les âges, est-ce qu'on dénonce, est-ce qu'on ne dénonce pas? (Danièle)

Enfin, on trouve dans les propos des répondant·e·s une logique qui fait appel à des arguments de type moral. Les jeunes femmes et hommes sont décrits comme des victimes d'une socialisation déficiente, de la pression du groupe et/ou des problèmes familiaux les amenant à considérer comme normal, voire à banaliser des comportements sexuels jugés déviants (sexualité précoce, sexualité de groupe, multiplication des partenaires, etc.). Le rôle professionnel consiste alors à leur faire prendre conscience des conséquences possibles de telles pratiques et de leur offrir d'autres modèles de sexualité. Les propos d'Alberto, conseiller en santé sexuelle dans un centre de prévention des IST destiné à la population hétérosexuelle et LGBT situé au Tessin, ne semblent pas dénoués des enjeux moraux.

Je suis quand même de l'avis que nous avons aussi la tâche de "promouvoir» une prise de conscience de la personne que nous suivons... sinon, il n'y aura jamais un changement, ni une attitude plus positive. (Alberto)

Ces arguments donnent lieu à des stratégies d'intervention caractérisées par l'orientation vers des spécialistes.

Des stratégies d'intervention axées sur l'orientation vers des spécialistes

Une lecture de la sexualité en termes hygiénistes, légalistes ou moralistes invite à considérer la sexualité comme «un monde à part», un champ d'action spécifique qui demande une expertise qui lui est propre pour pouvoir intervenir relevant, par exemple du champ médical ou juridique.

À un manque de formation pour pouvoir aborder ces aspects s'ajoute tabous et sentiments de malaise pour traiter les questions. Parfois, ces professionnel-le.s peuvent ressentir une tension entre leur souhait de ne pas adopter une posture jugeante dans leur intervention et la peur de le faire, malgré eux, ce qui peut paralyser l'intervention. Dans d'autres situations, tout en constatant le besoin des jeunes d'avoir un regard d'adulte au niveau de la sexualité, elles et ils se voient limités dans leurs actions face à la difficulté de concilier cette tâche avec la manière de comprendre leur rôle professionnel. En recourant à des arguments comme la neutralité ou l'importance de ne pas mélanger les rôles, elles et ils justifient leur position 
quant au fait de ne pas être les mieux placés pour aborder ces questions et la nécessité de déléguer ce traitement à des professionnel-le·s plus spécialisé·e·s (par exemple: psychothérapeutes, psychiatres, gynécologues, etc.). C'est ce qu'exprime Claudine, animatrice socioculturelle dans un centre de rencontres pour filles en Suisse romande. Elle argumente sa position par une préférence supposée des jeunes de parler des questions d'intimité avec une personne en dehors du cercle des relations quotidiennes.

Mais des fois, c'est hyper dur. Et puis jongler entre la confidence, le rapport de professionnelle qui dit: "Je ne suis pas ta sour ou ta copine mais en même temps, peut-être que je suis meilleure confidente que tes copines au final parce que je suis une adulte", c'est assez compliqué je trouve [...]. Parfois les ados, ce n'est pas à moi qu'ils veulent parler. Ils veulent parler à quelqu'un qui est encore plus en dehors [se réfère à une personne de planning familial], à qui ils peuvent se confier, mais sans le voir tous les jours ou toutes les semaines parce qu'ils ont aussi envie de garder une relation avec moi qui est telle ou telle. (Claudine)

Dans ces circonstances, la collaboration se présente comme une stratégie d'intervention privilégiée. Elle est comprise comme un ensemble d'expertises où chacun·e possède son propre domaine des compétences qu'il faut reconnaître et respecter et, en aucun cas, transgresser. Néanmoins, une fois l'information transmise, il n'y a plus de contrôle sur le suivi de la situation et certaines collaborations s'avèrent plus difficiles. Parfois, ces personnes peuvent ressentir une tension entre l'impression de ne pas avoir le choix de suivre les procédures, puisqu'elles ne sont pas elles-mêmes les expertes, et le constat que ces procédures ne s'avèrent pas forcément les solutions les plus adaptées pour répondre aux besoins des jeunes car les spécialistes n'apportent pas de réponses satisfaisantes. Enfin, cette stratégie d'orientation vers des spécialistes tend à écarter les questions de sexualité et de vie affective de leur travail socio-éducatif auprès des jeunes.

\section{Accompagner et développer des ressources des jeunes}

De l'autre côté du continuum, on trouve la posture professionnelle d'accompagnement. Les individus qui s'inscrivent dans cette logique considèrent l'éducation sexuelle, les enjeux et les problématiques qui peuvent en découler comme relevant d'une responsabilité partagée entre l'État (à travers l'école, les centres de prises en charge des jeunes, les mandats de santé sexuelle, etc.) et les parents. En effet, s'ils ou elles considèrent que l'intimité 
des jeunes doit être respectée, l'éducation sexuelle fait partie intégrante, à leurs yeux, de leur mission socio-éducative et elle ne doit pas être intégralement déléguée à des spécialistes.

Ces professionnel-le·s ont tendance à considérer les jeunes comme des actrices et acteurs possédant des ressources et étant capables de faire des choix. Les transactions sexuelles sont surtout considérées comme des nouvelles pratiques de sexualité qui peuvent choquer au regard d'une certaine morale, mais qu'il s'agit de ne pas dramatiser et de situer dans le contexte actuel des rapports entre jeunes. À ce titre, elles peuvent faire partie d'un parcours sexuel considéré comme «normal» dans le cadre de la socialisation sexuelle, visant l'exploration et l'affirmation de soi. Isabelle, conseillère en santé sexuelle, offre des consultations dans un canton en Suisse romande en la matière. Elle décrit les transactions comme une réalité assez présente dans la vie des jeunes.

Et puis dans ce qu'on discute, ce n'est pas si clair que ça et je pense qu'il y a aussi beaucoup de jeunes qui ne s'identifient pas du tout dans ce type d'échange parce que pour eux, ce n'est pas unéchange. Un mec qui a payé toute la journée ou le soir ou il a offert quelque chose et puis finalement, ça débouche sur ça. [...] Ce n'est pas forcément non plus toujours dramatique d'offrir une faveur sexuelle. (Isabelle)

Ces personnes décrivent leur rôle comme étant celui d'accueillir la parole des jeunes en leur offrant un espace d'écoute où ces dernières et derniers peuvent trouver par eux-mêmes des solutions et des réponses à des questions. Elles mettent une grande emphase sur la liberté de ces jeunes et la relativité des systèmes de valeurs. Leur intervention se fonde en priorité sur les besoins des jeunes.

Une légitimation centrée sur les besoins des jeunes

Ces professionnel-le·s considèrent que le fait de pouvoir parler de sexualité est un besoin des jeunes. La sexualité est comprise dans sa perspective holistique et ces personnes se sentent légitimes d'aborder des questions de sexualité dans le cadre de leur travail socio-éducatif. Elles abordent non seulement des aspects en lien avec la dimension biologique, mais s'intéressent aussi à l'identité, aux sentiments ou à l'orientation sexuelle des jeunes et, ceci, en profitant des différentes situations issues de la réalité du terrain (consultations, discussions informelles, etc.). Les intervenant.e.s insistent sur la nécessité d'être créatives et créatifs pour faire émerger des 
questions autour de la sexualité et d'avoir une attitude d'ouverture permettant aux jeunes de se sentir en confiance pour aborder des thématiques sensibles. Par exemple, Benoît, de par son expérience en tant que travailleur social scolaire et actuellement responsable d'un centre d'animation des jeunes situé en Suisse romande, utilise des occasions diverses telle que la distribution des préservatifs pour entamer la discussion sur le sujet.

C'est aussi parce qu'on distribue les préservatifs. Alors quand ils viennent nous demander "Je peux prendre deux préservatifs?», là on dit «Ok, t'as une copine? Super». Ils disent «C'est un peu chaud parce que je n'arrive pas à avoir une érec..., je suis un peu nerveux» des trucs comme ça. (Benoît)

Ces répondant·e·s mettent au centre de leur intervention la relation avec les jeunes et la création d'un lien de confiance. Elles et ils préconisent une écoute réflexive en sachant que des résultats (en termes de prévention) sont difficilement atteints à court terme.

Des stratégies d'intervention qui s'appuient sur les ressources des jeunes

Dans cette logique où les jeunes sont considérés comme étant dotés de ressources et possédant une grande capacité d'action, une importance capitale est donnée au respect de la diversité des pratiques, des valeurs et des rapports à la sexualité. Or, à trop miser sur les ressources des jeunes, cette logique peut parfois faire oublier que ces personnes sont encore en processus de construction identitaire et qu'elles peuvent avoir parfois besoin des adultes dans leur transition à la vie adulte. Cela peut donner lieu à des tensions d'ordre différent. Il peut y avoir une tension entre la peur d'adopter une posture trop normalisante, si elles et ils se réfèrent à des valeurs pour fonder leur intervention, et, à contrario, trop axée sur la relation qui les amènerait à relativiser les situations. C'est ce qu'exprime par exemple Mégane, infirmière scolaire dans un établissement situé en Suisse romande, qui reçoit plusieurs jeunes en consultation dans son bureau situé à l'école, pour des questions de sexualité.

Ily a quand même régulièrement des jeunes filles qui sont en formation duale ${ }^{6}$, donc elles sont en entreprise, qui ont des relations avec leur employeur. Et puis là, souvent elles sont majeures quand même. Elles ont au-delà de 16 ans. [...] Et donc ça avait fait un grand tollé dans l'école au niveau des adultes [...]. C'est vrai qu'en parlant avec des jeunes, il n'y avait pas de contrainte, cétait un moyen qu'ils utilisaient entre eux. Il n'y avait pas eu de violence et puis de 
mise sous contrainte, etc. Et puis des fois, c'est plus les valeurs des adultes qui sont bousculées, plus que les jeunes. Mais c'est vrai que moi je trouve toujours délicat les situations où il y a l'employeur qui a une relation avec l'apprentie, jusqu'à quel point elle est dominée, elle est sous contrainte. Je trouve délicat. (Mégane)

Ses propos montrent qu'une intervention d'accompagnement uniquement centrée sur la relation avec les jeunes offre peu de repères pour décoder les souffrances tacites, juger du caractère problématique ou non de telles situations et guider leurs pratiques. D’ailleurs, il semblerait y avoir une confusion entre le "non-jugement» des jeunes et le «non-jugement» des situations, pouvant amener les intervenant·e·s à douter de leur légitimité à les orienter ou à leur offrir de l'aide, alors que ces jeunes peuvent en formuler la demande, de manière plus ou moins directe.

Cette approche se veut critique face à des actions fondées sur des visions trop normatives de la sexualité (cherchant à cadrer et contrôler la sexualité des jeunes à partir de normes morales, hygiénistes et/ou légalistes). Or, les personnes interviewées ont le sentiment de défendre une approche à contre-courant, qui n'est pas toujours reconnue, voire soutenue par leurs directions ou leurs collègues, ce qui peut rendre leur tâche plus difficile. Leur posture peut rencontrer des résistances face aux individus qui privilégient une approche de protection des jeunes, car elles peuvent être perçues comme intrusives par rapport à des sujets qui relèvent de la responsabilité privée, ou alors comme développant des modes d'intervention qui ne protègent pas suffisamment ou pas assez rapidement les jeunes, voire comme encourageant des comportements considérés comme déviants. C’est ce que décrit Mégane. En tant qu'infirmière scolaire, elle connaît bien les contraintes liées au cadre scolaire qui empêchent une promotion du thème de la sexualité à travers des espaces collectifs, par exemple, les journées à thèmes.

Après, c'est très règlementé au niveau scolaire avec qui on peut travailler [...]. Chaque direction peut mettre son veto en fonction des intervenants. Il y a des thèmes-sujets qui sont sensibles pour certaines directions [...]. Donc des fois, c'est compliqué quand on est seule dans un établissement à promouvoir ce thème-là, ce côté-là où je pense qu'on pourrait vraiment se rendre visible et puis accessible par rapport à ce thème-là. [...] Les journées à thème ou les moments de stands d'information fonctionnent bien auprès des élèves. [...] Les jeunes en ont besoin. Parler différemment autour de ces thèmes-là pas 
que dans l'intimité. Je pense que ça les interpelle différemment d'être en collectif. (Mégane)

Enfin, ces personnes ne s'opposent pas à orienter les jeunes à des collègues lorsque la situation le nécessite, mais la collaboration est alors comprise comme la création de liens et un travail de réseautage qui implique une connaissance réciproque, des moments d'intervision, de débriefing, etc. En effet, elles craignent parfois que la référence à des spécialistes soit interprétée par les jeunes comme une trahison de la confiance établie avec elles et eux. C'est pourquoi elles accordent de l'importance à bien connaître le réseau professionnel et veillent, en cas de transfert vers d'autres spécialistes, à bien préparer ce passage avec la ou le jeune. Fiona, médiatrice scolaire dans un établissement en Suisse romande, propose, dans le cadre de son activité professionnelle, des séances d'écoute aux élèves et participe aux projets de promotion et de prévention de santé. Pour elle, l'orientation vers une autre personne demande l'établissement d'une relation de confiance autant avec la ou le professionnel-le en question qu'avec la ou le jeune concerné.

À l'école, on a eu un moment une infirmière et clairement, les élèves n'allaient pas lui parler de sexe. Donc clairement, je ne vais pas pouvoir les envoyer chez elle. C'était du style elle retenait presque en otage les brochures VoGay [organisme de soutien aux personnes homosexuelles] [...] Il y a des difficultés comme ça qui sont de terrain [...] on trouve tous un peu des stratégies [...] Quand un jeune vient vers moi et puis que j'établis la relation de confiance et je lui dis: "Maintenant, tu vois, je ne suis pas compétente mais j'ai vraiment envie que tu puisses bénéficier de l'aide dont tu as besoin, je vais t'accompagner chez quelqu'un", il faut que je puisse garantir que cette personne, elle soit à la hauteur de la confiance... (Fiona)

\section{Conclusion}

Le graphique ci-dessous résume le continuum entre les deux postures professionnelles qui ressortent des entretiens auprès des professionnelles et professionnels, avec les représentations sur lesquelles elles s'appuient (de la sexualité d'ordre transactionnel et des jeunes qui s'y engagent), leurs légitimations et les stratégies professionnelles qui en découlent. 


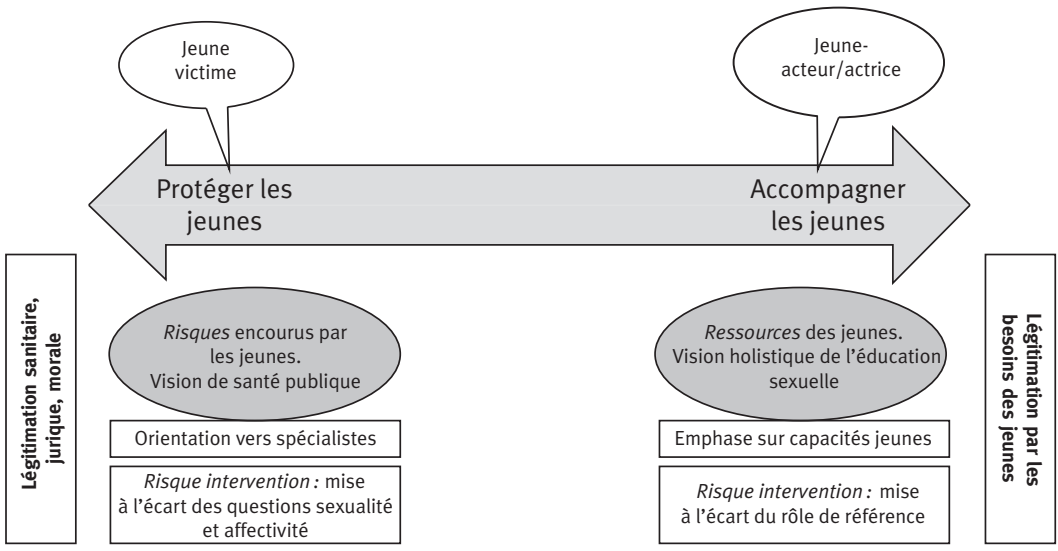

Source: "Sexe, relations... et toi? » Sexualité et transactions sexuelles impliquant des jeunes. Résultats du volet 3 : focus groups auprès de professionnel-le·s du travail social et de la santé.

Les résultats présentés dans cet article montrent que plusieurs professionnel-le.s se sentent peu, voire pas légitimes d'aborder les questions d'éducation sexuelle au sein de leur travail auprès des jeunes. Elles ou ils sont plusieurs à privilégier davantage une posture de protection qui favorise la délégation de l'éducation sexuelle à des spécialistes. Même celles et ceux qui s'inscrivent davantage dans une posture d'accompagnement ne savent pas toujours comment aborder les questions de sexualité avec les jeunes.

Deux processus expliquent ce sentiment d'illégitimité. Premièrement, comme le constatent également d'autres études, la plupart des professionnel-le-s interviewé.e.s dans le cadre de cette étude semblent être des «héritiers d'une tradition qui avait fait de la question sexuelle un objet relevant d'une dimension sanitaire; et peu ou pas pensée dans sa ‘dimension globale`» (Amsellem-Mainguy \& Dumollard, 2016, p. 12). Ainsi, bien que les ancrages sanitaires et sociaux de la sexualité soient davantage reconnus au sein du travail social, les professionnel-le.s de ce champ peinent à intégrer concrètement une approche holistique de la sexualité dans leur intervention auprès des jeunes.

Deuxièmement, comme le montrent également d'autres études, l'éducation sexuelle est encore très peu intégrée à la formation des travailleuses et travailleurs sociaux, ce qui peut également contribuer à renforcer l'idée que ces questions ne peuvent être traitées que par des spécialistes. 
En effet, parmi les participant·e·s à notre enquête, ce sont principalement celles et ceux ayant une formation sociale qui adhèrent à la posture professionnelle de protection, alors que les répondant·e·s ayant une formation santé ou social et possédant (dans la plupart des cas) des diplômes postgrades en santé sexuelle ont tendance à adhérer davantage à la posture professionnelle d'accompagnement. Ces résultats laissent penser qu'une formation spécifique à la sexualité contribue à légitimer l'intégration de l'éducation sexuelle dans le travail socio-éducatif auprès des jeunes.

$\mathrm{Au}$ terme de cet article, il nous semble intéressant de mettre ces résultats en perspective avec ceux des autres volets de l'étude qui visaient à saisir les représentations que les jeunes ont des transactions sexuelles. Du point de vue des jeunes, les expériences de transactions sexuelles ne sont pas en soi problématiques. Au contraire, certaines peuvent contribuer à construire l'intimité entre pairs nécessaire à la construction identitaire et à l'autonomie sexuelle. Or, même si la sexualité semble débarrassée des tabous sociaux dont elle faisait l'objet, elle reste un sujet sensible et associé à des attentes de comportements très codifiées, notamment selon le genre (Colombo et al., 2017b; Carbajal et al., 2019). Dans ce contexte, les jeunes se posent des questions sur leur sexualité, leur vie affective et leurs relations amicales et elles et ils attendent des intervenant.e.s socio-sanitaires qu'elles et ils les aident à répondre à ces questions.

Par ailleurs, si les expériences de transactions sexuelles ne sont pas en soit problématiques, elles peuvent toutefois le devenir lorsqu'elles s'inscrivent dans des rapports de force inégaux, qui peuvent nécessiter l'intervention d'adultes. Or, certain·e·s jeunes rencontré·e·s nous ont fait part de leur difficulté à parler de ces situations, de peur de se voir jugé·e·s par des professionnel.le·s qui, parfois sans le vouloir, peuvent renforcer les normes de genre liées au système hétéronormatif, laissant peu (ou pas) de place à des identités de genre ou à des orientations sexuelles différentes.

Ces résultats renforcent l'idée que l'éducation sexuelle ne peut pas être uniquement déléguée à des spécialistes, mais qu'elle doit être mieux intégrée à la mission socio-éducative de travail social. Or, pour ce faire, il apparaît nécessaire de renforcer la légitimité de la place de l'éducation sexuelle au sein de ce métier, notamment en développant la formation de base et continue des travailleuses et travailleurs sociaux dans ce domaine. Nos résultats indiquent en particulier trois axes sur lesquels cette offre de formation pourrait prendre appui. Un premier axe est la sensibilisation à une éducation sexuelle holistique, qui donne des outils pour aborder la sexualité à partir de différents aspects de la vie des jeunes (défense de leurs 
droits, questions liées au respect, au consentement, etc.). Le deuxième axe vise à former, informer et soutenir ces professionnel-le·s par rapport aux questions de vie affective et sexuelle des jeunes. Cela veut dire les outiller pour décoder les signaux envoyés par les jeunes dans différents contextes (changements de comportement, allusions indirectes, etc.) et leur donner des pistes concrètes pour mieux se positionner et savoir comment répondre aux questionnements et problématiques des jeunes et vers qui les diriger lorsque cela dépasse leur mandat. Finalement, un troisième axe concerne la sensibilisation à la diversité des manières d'incarner les identités de genre et de vivre sa vie sexuelle et affective, de manière à combattre les stéréotypes et représentations sociales qui peuvent contribuer à reproduire des rapports de pouvoir inégalitaires et décourager les jeunes de demander de l'aide, de peur de se sentir stigmatisé·e·s (Colombo \& Carbajal, 2018).

\section{Annexe 1}

Tableau 1:

Participant·e.s aux focus groups ${ }^{a}$

\begin{tabular}{|c|c|c|c|}
\hline Prénom d'emprunt & Institution & Profession & Région linguistique \\
\hline Eric & Action Educative en Milieu Ouvert (AEMO) & Éducateur social & Suisse romande \\
\hline Gaëlle & Association défense travail du sexe & $\begin{array}{l}\text { Aide-soignante dans le médico- } \\
\text { social, sexologue }\end{array}$ & Suisse romande \\
\hline Sandro & Brigade des mœurs ${ }^{b}$ & Policier & Suisse italienne \\
\hline Gérard & Brigade des mœurs ${ }^{b}$ & Policier & Suisse romande \\
\hline Claudine & Centre d'animation & Animatrice socio-culturelle & Suisse romande \\
\hline Benoît & Centre d'animation & Animateur socio-culturel & Suisse romande \\
\hline Fabienne & Centre d'animation & Animatrice socio-culturelle & Suisse romande \\
\hline Louise & Centre d'animation & Animatrice socio-culturelle & Suisse romande \\
\hline Yvonne & Centre d'animation & Animatrice socio-culturelle & Suisse romande \\
\hline Jeanne & Centre d'animation & Animatrice socio-culturelle & Suisse allemande \\
\hline Bertrand & $\begin{array}{l}\text { Centre de consultations en santé sexuelle } \\
\text { public LGBT }\end{array}$ & Conseillère en santé sexuelle & Suisse romande \\
\hline Olivier & $\begin{array}{l}\text { Centre de consultations en santé sexuelle } \\
\text { public LGBT }\end{array}$ & Conseillère en santé sexuelle & Suisse romande \\
\hline Camille & Centre de consultations en santé sexuelle & Conseillère en santé sexuelle & Suisse romande \\
\hline Bianca & Centre de consultations en santé sexuelle & Conseillère en santé sexuelle & Suisse italienne \\
\hline Alberto & $\begin{array}{l}\text { Centre de consultations en santé sexuelle } \\
\text { public LGBT }\end{array}$ & Conseillère en santé sexuelle & Suisse italienne \\
\hline Isabelle & Centre de consultations en santé sexuelle & Conseillère en santé sexuelle & Suisse romande \\
\hline Sophie & Hôpital, Service de gynécologie & Gynécologue & Suisse romande \\
\hline Barbara & $\begin{array}{l}\text { Consultations et soutien psychologiques/ } \\
\text { Ligne d'aide }\end{array}$ & Travailleuse sociale & Suisse allemande \\
\hline Norbert & $\begin{array}{l}\text { Consultations et soutien psychologique/ } \\
\text { Ligne d'aide }\end{array}$ & Psychologue & Suisse romande \\
\hline Fiona & École & Médiatrice scolaire & Suisse romande \\
\hline Mégane & École & Infirmière scolaire & Suisse romande \\
\hline
\end{tabular}


Continuation du tableau 1.

\begin{tabular}{llll}
\hline Prénom d'emprunt & Institution & Profession & Région linguistique \\
\hline Karla & École & Travailleuse sociale scolaire & Suisse allemande \\
Patrice & École & Travailleur social scolaire & Suisse romande \\
Jade & École & Travailleuse sociale scolaire & Suisse romande \\
Urs & Éducation sexuelle & Éducateur en santé sexuelle & Suisse allemande \\
Marion & Éducation sexuelle & Éducatrice en santé sexuelle & Suisse allemande \\
Francesco & Fondation Protection jeunesse & Cadre en travail social & Suisse italienne \\
Lea & Foyer jeunes en difficulté & Éducatrice sociale & Suisse romande \\
Matteo & Foyer jeunes en difficulté & Psychologue & Suisse italienne \\
Annika & Foyer jeunes en difficulté & Enseignante & Suisse allemande \\
René & Service communal de jeunesse & Travailleur social & Suisse romande \\
Massimo & Service communal de jeunesse & Éducateur social de rue & Suisse italienne \\
Danièle & Service Protection de la Jeunesse & Assistante sociale & Suisse romande \\
Patricia & Service Protection de la Jeunesse & Assistante sociale & Suisse romande \\
\hline
\end{tabular}

${ }^{a}$ Nous avons intégré dans cette liste une répondant·e qui ne pouvait pas participer aux focus groups et que nous avons rencontré-e en entretien individuel.

${ }^{\mathrm{b}}$ Afin de compléter le panorama, 2 policiers de la Brigade des mœurs ont été également invités à participer aux deux focus groups, mais leurs propos ne sont pas traités dans le cadre de cet article.

\section{Références bibliographiques}

Amsellem-Mainguy, Yaëlle \& Dumollard, Marie (2016). Dits et non-dits sur la sexualité dans la relation entre jeunes et professionnels de la Protection Judiciaire de la Jeunesse. Champ pénal / Pénal field [En ligne], 13, https://doi. org/10.4000/champpenal.9311

Bauer, Michael, Haesler, Emily \& Fetherstonhaugh, Deirdre (2015). Let's talk about sex: older people's views on the recognition of sexuality and sexual health in the health-care setting. Health Expectations, 19, 1237-1250.

Broqua, Christophe \& Deschamps, Catherine (2014). Transactions sexuelles et imbrications des rapports de pouvoirs. In: Christophe Borqua \& Catherine Deschamps (Éds.). L'échange économicosexuel, p. 7-18. Éditions de l'École des hautes études en sciences sociales.

Carbajal, Myrian, Colombo, Annamaria \& Tadorian, Marc (2019). Consentir à de relations sexuelles sans en avoir envie. La logique de la redevabilité: responsa- bilité individuelle ou injonction sociale genrée? Journal des anthropologues, 156-157, 197-218.

Charmillot, Maryvonne \& Jacot-Descombes, Caroline (2018). Penser l'éducation sexuelle à partir des droits sexuels. Recherches \& éducations [En ligne], https://doi.org/10.4000/rechercheseducations.6758

Combessie, Philippe \& Mayer, Sybilla (2013). Une nouvelle économie des relations sexuelles. Ethnologie française, 43 (3), 381-389.

Colombo, Annamaria, Pulzer, Noémie \& Parazelli, Michel (2016). Représentations sociales des sans-abri. La mobilisation des Enfants de Don Quichotte à Paris. Déviance et Société, 40 (1), 51-77.

Colombo, Annamaria, Carbajal, Myrian, Carvalhosa Barbosa, Marlène, Jacot, Cédric \& Tadorian, Marc (2017a). Sexe, relations... et toi? Sexualité et transactions sexuelles impliquant des jeunes. Synthèse des résultats de recherche. HES-SO, Haute école de travail social 
Fribourg (HETS-FR), Fondation Oak. Fribourg: HETS-FR. Récupéré de: www. sexe-et-toi.ch ou www.hets-fr.ch

Colombo, Annamaria, Carbajal, Myrian, Carvalhosa Barbosa, Marlène \& Tadorian, Marc (2017b). Gagner la reconnaissance des pairs en évitant la réputation de "pute». L'injonction paradoxale qui pèse sur les filles impliquées dans des transactions sexuelles. Revue Jeunes et Société, 2(2), 70-93.

Colombo, Annamaria \& Carbajal, Myrian (2018). Sexe, relations... et toi?

Recommandations. Récupéré de: https://2238aeba-82b6-4d2a-9a78-dfbccbf074dc.filesusr.com/ugd/1f0318 d6ba3a422720404b8e5a84d0dabdc095. pdf

Côté, Philippe-Benoit, Manseau, Hélène, Boucher, Kathleen, Provencher, MarieAndrée \& Blais, Martin (2015). Les interventions en matière de sexualité chez les jeunes en situation de rue à Montréal: l'accompagnement social comme piste prometteuse. Revue canadienne de service social, 32 (1-2), 95-111.

Darnaud, Thierry, Sirvain, Serge, Valérie lgier, Taiton, Martine (2013). A study of hidden sexuality in elderly people living in institutions. In: Sexologies, 22, p. $169-175$.

Dodd, Sarah-Jane \& Tolman, Deborah (2017). Reviving a Positive Discourse on Sexuality within Social Work. Social Work, 62 (3), 227-234.

Giami, Alain (2009). Les formes contemporaines de la médicalisation de la sexualité. In Sanni Yaya (Éd.). Pouvoir médical et santé totalitaire: conséquences socio-anthropologiques et éthiques, p. 225-249. Presses Université de Laval.

Giami, Alain, Moulin, Pierre \& Moreau, Emilie (2013). La place de la sexualité dans le travail infirmier: l'érotisation de la relation de soins. In: Varia, 55 (1), 20-38, https://doi.org/10.4000/sdt.6243

Giami, Alain (2018). Les soignants face à l'intimité et à la sexualité, SOiNS, 831, 44-47. HES-SO, 2020. Plan d'étude cadre 2020. Bachelor of Arts HES-SO en Travail social. Récupéré de: https://www.hes-so. ch/fr/plan-detudes-cadre-2020-bachelor-arts-hes-so-15800.html

Jodelet, Denise (1989). Représentations sociales: Un domaine en expansion. In: Denise Jodelet (Éd.), Les représentations sociales, p. 47-78. PUF.

Joël, Myriam (2014). La gestion de la sexualité entre femmes et hommes détenus, à partir de l'étude des prisons de femmes. VST (vie social et traitements), 4 (124), 28-34.

Karsz, Saül (2004). Pourquoi le travail social? Définition, figures, clinique. Paris: Dunod.

Krueger, Richard A. (1998). Moderating Focus Groups. Focus Group Kit 4. Thousand Oaks: Sage.

McCave, Emily, Shepard, Benjamin \& Ramseyer Winter, Virginia (2014). Human Sexuality as a Critical Subfield in Social Work. Advances in Social work, 15 (2), 409-427.

Ménoreau, Jean Siméon \& Dupras, André (2014). Accompagner la sexualité des personnes ayant une déficience intellectuelle: l'apport d'un changement institutionnel et d'une formation professionnelle. Sexologies, 23, 173-178.

Merotte, Lisbeth (2012). La sexualité en détention : analyse de trois méthodes d'investigation. Sexologies, 21, 157-160.

Morton, Julie, Jeyasingham, Dharman \& Hicks, Stephen (2013). The Social Work of Sexuality: Rethinking Approaches to Social Work Education. Health and Social Care Education, 2 (2), 16-19.

Moulin, Pierre (2007). La construction de la sexualité chez les professionnels de santé et du travail social ou la normalisation des conduites profanes. ERES "Nouvelle revue de psychosociologie", 2 (4), 59-88.

OMS Bureau régional pour l'Europe et BZgA (2010/2013 version française), Standards pour l'éducation sexuelle en Europe. Lausanne: Santé sexuelle suisse. Récupéré de: https://www.alliance-educationsexuelle.ch/jt_files/jt_files_filename_0005_1202974180.pdf 
Rowntree, Margaret R. (2014). Making Sexuality Visible in Australian Social Work Education. Social Work Education, 33 (3), 353-364.

Schaub, Jason, Willis, Paul \& Dunk-West, Priscilla (2017). Accounting for Self, Sex and Sexuality in UK Social Workers' Knowledge Base: Findings from an Exploratory Study. British Journal of Social Work, 47, 427-446.

SEFRI Secrétariat d'État à la formation, à la recherche et à l'innovation (2020). Système éducatif suisse. Récupéré de: https://www.sbfi.admin.ch/sbfi/fr/ home/formation/l-espace-suisse-de-formation/systeme-educatif-suisse.html

Tabet, Paula (2004). La grande arnaque. Sexualité des femmes et échange économico-sexuel. L'Hamattan.

Taylor, Abi \& Gosney, Margot A. (2011). Sexuality in older age: essential considerations for healthcare professionals. Age and Ageing, 40, 538-543.

Zelizer, Viviana (2001). Transactions intimes. Genèses, 42 (1), 121-144.

\section{Annotations}

1 Prénom d'emprunt. Dans la suite de l'article, nous allons présenter le profil de la personne enquêtée dans le texte et non à la suite de ses propos.

2 Dans le cadre de cet article nous utilisons de manière indistincte les termes «professionnel-le» et «intervenant.e».

3 OMS, Santé sexuelle (consulté le 1.03.2021). Récupéré de: https://www. who.int/topics/sexual_health/fr/

4 Mentionnons, à titre d'exemple, les travaux de Giami sur la pratique infirmière et l'érotisation de la relation des soins (Giami et al., 2013; Giami, 2018), ceux sur la sexualité des personnes âgées et atteintes de la maladie d'Alzheimer (Taylor et al., 2011; Giami/Ory, 2012; Darnaud et al., 2013; Bauer et al., 2015) ou encore ceux abordant le malaise des membres du corps médical pour traiter des questions de sexualité (Merotte, 2012 entre autres).

5 En raison du nombre limité de signes, cet article se concentre sur la place de l'éducation sexuelle dans la relation socio-éducative et met moins l'accent sur la prégnance des stéréotypes et normes de genre dans les représentations sociales et pratiques d'intervention des professionnel-le.s, qui fera l'objet d'une autre publication.

6 Le système suisse de formation duale ou en alternance, qui est la plus répandue dans le pays, permet aux jeunes ayant fini l'école obligatoire de se former dans une entreprise tout en suivant des cours dans une école professionnelle (SEFRI 2014).

\section{Notes biographiques}

Myrian Carbajal est docteure en sciences sociales et professeure à la Haute école de travail social Fribourg, Haute école spécialisée de Suisse occidentale (HES-SO). Elle est spécialisée dans les questions de migration, de sexualité et de genre. Elle s'intéresse aux logiques et capacités d'action des individus. Email: myrian.carbajal@hefr.ch.
Annamaria Colombo est docteure en sciences sociales et professeure à la Haute école de travail social Fribourg, Haute école spécialisée de Suisse occidentale (HES-SO). Elle est spécialisée dans le champ des marginalités urbaines et juvéniles. Ses recherches s'intéressent aux enjeux liés à la reconnaissance, à la socialisation et aux comportements dits à risque. Email: annamaria.colombo@hefr.ch 